\title{
Wideband Digital Phase Shifter with Low Insertion Loss
}

\author{
Chengpeng Liu ${ }^{a}$, Zhengrong $\mathrm{He}^{\mathrm{b}}$ \\ Sichuan Institute of Solid State Circuits China Electronics Technology Group Corp, Chongqing \\ 400060, China. \\ alcp54913@163.com, bhzr73525@sina.com
}

\begin{abstract}
In this paper, we first analyze the structure of the commonly used phase shifter and analyze its characteristics. Secondly, after choosing the high-pass and low-pass filter structure to design the phase shifter, the characteristics of the phase shifter are analyzed and selected respectively., The simulation results meet the design requirements. The circuit has been through $0.5 \mu \mathrm{m}$ GaAs process, simulation tools for ads2011. The phase shifter designed over the entire operating frequency band exhibits good performance.
\end{abstract}

Keywords: Wideband; MMIC; phase shifter; insertion loss.

\section{Introduction}

With the rapid development of military and civilian technology, phase shifter has been more and more widely used, its basic purpose is to change the phase of an input signal we need a phase angle, we can change the phase shifter work Different states, by controlling the switching device on, off to achieve, so as to obtain a different amount of phase shift. There are two kinds of phase shifter, one is the digital phase shifter, its phase shift in the way of change in accordance with a certain step to jump-type changes. The other is the analog phase shifter, its input control signal is an analog signal, so its phase shift is a continuous process of change [1].

In the case of a digital phase shifter, the accuracy is largely determined by its number of bits. The more bits there are, the more the status bits are, the shorter the corresponding step size, and the more accurate phase shifts. At the same time, the circuit design will be a lot of complexity, the occupied area of the chip will become larger.

Digital phase shifter is relatively faster than the analog phase shifter, the circuit is relatively simple, take up chip area is small, and so its modern communication systems have been widely used.

In the military and civil fields, CNC phase shifter as a very important part of the existence of mobile phones, personal communication satellite network, GPS, direct broadcast satellite reception, some tactical missiles, advanced phased array radar and so on. As an important part of a $\mathrm{T}$ \} component, the numerical control phase shifter will directly affect the performance of the CNC phase shifter and affect the whole system reliability index. Such as its phase-shifting step size, phase-shifting accuracy, slew rate in different states, reliability and stability, and so on. Therefore, a good performance of the CNC phase shifter for military missiles, radar or civilian instrumentation plays an important role, is an integral part of $[2,3]$.

\section{Phase Shifter Structure}

In the phase-shifting circuit design, any reactive element in series or in parallel with the transmission line introduces a phase shift, which makes the circuit as a phase-shifted topology a lot. However, in the actual application of the circuit, we need to consider some other performance requirements, such as the phase shifter insertion loss and port reflection loss are small, small phase shift and so on. Therefore, the actual circuit after the preferred form is not much. The following are some typical phase shifter structures $[4,5]$ :

(1) Switching linear phase shifter

Switching linear phase shifter, as shown in Figure 1, we can see, the application circuit switch control input signal, when coupled with a certain control voltage, the input signal through the two branches can be transmitted separately, the two The electrical length of each branch has a constant 
difference of $\Delta$. Because of such a difference, our circuit obtains two different phase states. For this circuit, we assume that the circuit is composed of ideal components Device combinations. At this point, the input signal from the conduction branch of the transfer, the two different phase state of the phase shift $\Delta$ always change with the $\Delta$, the value of its direct decision, because the phase shift is with the frequency While the linear change, it is also known as the dispersion-type phase shifter.

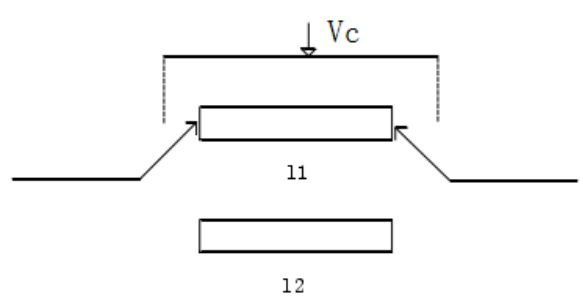

Fig. 1 Switching Linear Phase Shifter

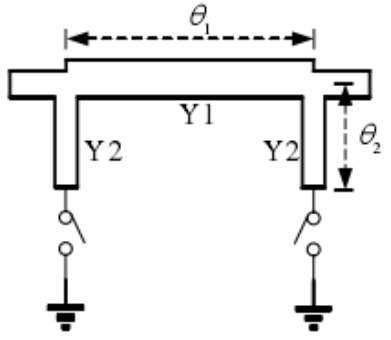

Fig. 2 Load lLinear Phase Shifter

(2) Load linear phase shifter

Load linear phase shifter is the basic principle of switching through the switch to change the reactance of the transmission network, in order to achieve the phase shift between different states of movement. Figure 2 shows a load-line phase shifter circuit model. The circuit comprises a main transmission line part and an admittance switching part, the main transmission line is a microstrip transmission line, and the admittance switching part is composed of parallel microstrip lines and switches at both ends of the main transmission line. When the switch is turned on and off, the phase shifters are equivalent to different lengths of transmission lines, the phase shift of the phase shifter is the difference between the two.

(3) Reflection type phase shifter

The basic principle of the reflection phase shifter is to connect the reactive load to the terminal on the uniform transmission line. The switching element can adjust the impedance of the load and adjust the phase of the reflection coefficient of the load, so that the Resulting in a certain phase shift.

Reflective phase shifter transmission line terminal can be divided into two categories: one is the use of the form of reactor network terminal, the switch is turned off and closed the moment, the entire circuit network input impedance will have a corresponding change, which is a commonly used form of circuit, which we call a switched reactance type. This type of reactance network is usually designed with a microstrip network, and capacitors and inductor devices can be used at lower frequencies. The other is the use of single-pole single-throw switch to achieve, after the switch with a short-term terminal of the transmission line, which is linear with the switching phase shifter works very similar, when the circuit switch is not connected, the signal Transmission line transmission to reach the short-circuit point will be reflected back again when the entire circuit phase change for the short-circuit transmission line electrical length twice, so it is compared to the switching line-type phase shifter required for the transmission line as long as the original two .

(4) High-pass, low-pass phase shifter
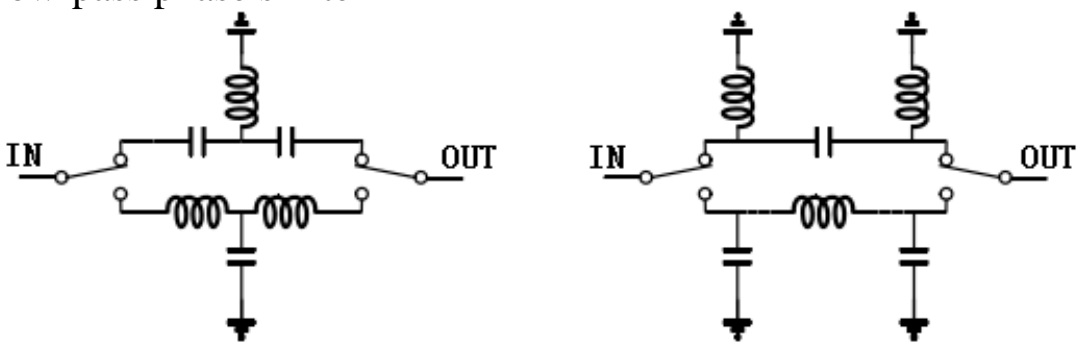

Fig. 3 High-pass, Low-pass Phase Shifter

High and low-pass phase shifter can be composed of lumped components, the advantages of lumped components is relatively smaller than the size of the electromagnetic signal through the signal is relatively small, it can be made into a very compact broadband phase shifter, and the actual On the main decision of the entire phase shifter circuit chip size is part of the switch circuit. Therefore, this 
circuit is very advantageous, especially in today's high integration requirements of the single-chip case.

High-pass, low-pass phase shifter, in the two branches of the circuit were access to T-type high-pass and low-pass filter, the former produce a leading phase, which produces a backward phase, so the two states Also produces a certain value between the phase difference. This circuit is characterized by two different spectral response circuit, high-pass circuit with the frequency of the advance phase will always be reduced, and low-pass circuit hysteresis phase will increase as the frequency increases, for these two filters can be mutually compensated for their phase variations. By combining these two filters, we can obtain a relatively flat phase-shift frequency response over a wide range of operating frequencies, and such circuits are particularly suitable for Monolithic broadband phase shifter.

\section{Circuit Design}

At the beginning of the design, should first be based on specific needs, select the most appropriate phase shifter structure. The digital phase shifter designed in this paper adopts high-low-pass phase-shifting network. High-low-pass phase shifter can achieve a flat phase-shift frequency response in a relatively wide frequency band. The high-low-pass phase shifter replaces the transmission line with a phase-shifted network, and uses a lumped element to facilitate monolithic integration. The whole network is mainly composed of FET switches, spiral inductors, MIM capacitors and other components.

Second, each basic bit design, should be based on multi-function MMIC phase shifter on the overall target requirements, specific to each basic bit design. Again, in the basic bit design process, should pay attention to the principle of complementary circuit design, the overall design error to a minimum. Finally, consider the problem of high and low temperature stability of the circuit, under normal circumstances, the overall gate-to-gate ratio of small FET transistors on the temperature changes are more sensitive, therefore, should be selected in the design of the total gate FET transistor.

Each phase shifter has two states: the reference state and the phase-shifted state. The switching between the two states is achieved by turning on and off the transistor. Select the appropriate cascade sequence to cascade the basic phase shift, generally follow the principle: the standing wave of good basic bits on both ends, so you can easily get a good input / output port standing wave. Select the appropriate matching microstrip line to cascade all the basic bits, you can get a complete digital control phase shifter.
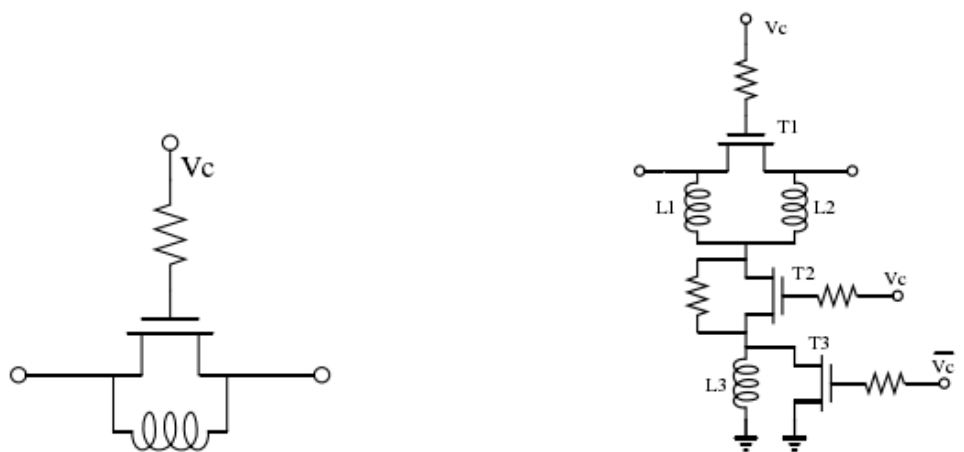

Fig. 4 simplified high-low pass phase shift

45-degree, 90-degree and 18-degree phase shifter circuit topology using T-type high-low-pass phase-shift network, this structure can be considered from the switching linear phase shifter from the use of phase-shifting network instead of transmission lines. When the signal is switched from the network, a different phase is obtained, resulting in a phase shift. The main advantage is that it is possible to design a phase shift that varies with frequency, to obtain a wider frequency band and a better frequency response. The use of series-parallel switch connection can improve the isolation of the switch to reduce the choice of different paths to other path phase interference. 
5.625 degrees, 11.25 degrees and 22.5 degrees phase shifter circuit topology using a simplified high-low pass phase shift network.

\section{Simulation Results}

In this paper, the main research for the digital phase shifter based on foundry technology, so the use of MMIC technology design has been realized by $0.5 \mu \mathrm{m}$ GaAs process. This digital phase shifter exhibits high performance. The simulation of our mixer have been presented based on the ADS. over 2.1 2.7GHz the proposed circuit conversion phase shift characteristics shown in Figure 5, to meet the design requirements. The insertion loss for all phase-shifted states is less than $6.3 \mathrm{~dB}$ over the entire operating frequency band

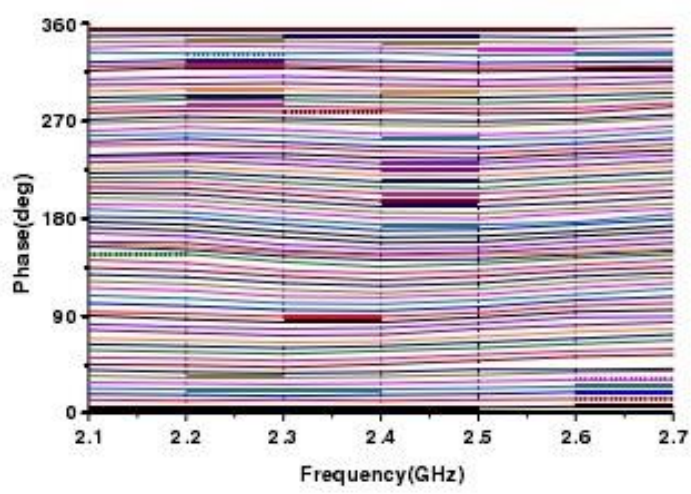

Fig. 5 All phase shift

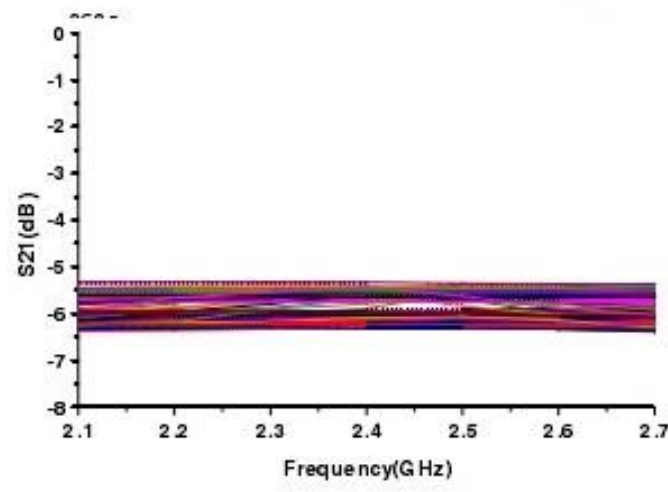

Fig. 6 Insertion loss

\section{Conclusion}

In this paper, we first analyze the structure of the commonly used phase shifter and analyze its characteristics. Secondly, after choosing the high-pass and low-pass filter structure to design the phase shifter, the characteristics of the phase shifter are analyzed and selected respectively. Finally, we pay attention to the particularity of phase shifter design, simulation results to meet the design requirements.

\section{References}

[1] Vaucher A M, Striffler C D, Lee C H. Theory of optically controlled millimeter-wave phase shifters [J]. Microwave Theory and Techniques, IEEE Transactions on, 1983, 31(2): 209-216.

[2] Campbell C F, Brown S A. A compact 5-bit phase-shifter MMIC for K-band satellite communication systems [J]. Microwave Theory and Techniques, IEEE Transactions on, 2000, 48(12): 2652-2656.

[3] Kwang-Jin Koh, Gabriel M. Rebeiz. 0.13um CMOS Phase Shifters for X-, Ku-, and K-Band Phased Arrays [J]. IEEE Journal of Solid-State Circuits. 2007, 42(11):2535-2546.

[4] Bahl I J, Conway D. L-and S-band compact octave bandwidth 4-bit MMIC phase shifters [J]. Microwave Theory and Techniques, IEEE Transactions on, 2008, 56(2):293-299.

[5] Yong sheng D, Tang sheng C, Li jie Y, et al. A novel multi-octave 22.5 degree and 11.25 degree MMIC phase shifter with a sharing Lange coupler [J]. Journal of Functional Marterial and Devices, 2000, 6(3): 137-140. 